\title{
Target-object integration, attention distribution, and object orientation interactively modulate object-based selection
}

\author{
Shahd Al-Janabi ${ }^{1}$ • Adam S. Greenberg ${ }^{1}$
}

Published online: 19 May 2016

(C) The Psychonomic Society, Inc. 2016

\begin{abstract}
The representational basis of attentional selection can be object-based. Various studies have suggested, however, that object-based selection is less robust than spatial selection across experimental paradigms. We sought to examine the manner by which the following factors might explain this variation: Target-Object Integration (targets 'on' vs. part 'of' an object), Attention Distribution (narrow vs. wide), and Object Orientation (horizontal vs. vertical). In Experiment 1, participants discriminated between two targets presented 'on' an object in one session, or presented as a change 'of' an object in another session. There was no spatial cue-thus, attention was initially focused widely - and the objects were horizontal or vertical. We found evidence of object-based selection only when targets constituted a change 'of' an object. Additionally, object orientation modulated the sign of objectbased selection: We observed a same-object advantage for horizontal objects, but a same-object cost for vertical objects. In Experiment 2, an informative cue preceded a single target presented 'on' an object or as a change 'of' an object (thus, attention was initially focused narrowly). Unlike in Experiment 1, we found evidence of object-based selection independent of target-object integration. We again found that the sign of selection was modulated by the objects' orientation. This result may reflect a meridian effect, which emerged due to anisotropies in the cortical representations when attention is oriented endogenously. Experiment 3 revealed that object orientation did not modulate object-based selection when attention was oriented exogenously. Our findings suggest that
\end{abstract}

Adam S. Greenberg

agreenb@uwm.edu

1 Department of Psychology, University of Wisconsin-Milwaukee, Milwaukee, WI 53201, USA target-object integration, attention distribution, and object orientation modulate object-based selection, but only in combination.

Keywords Object-based attention - Space-based attention . Meridian effect

Visual attention serves to filter irrelevant inputs from relevant inputs. This process can be accomplished through the prioritization of important locations (see, e.g., Posner \& Cohen, 1984) or important objects (see, e.g., Duncan, 1984) in the visual field. These forms of selective attention (space- and object-based, respectively) often work in tandem, as has been evidenced by studies that utilize the double-rectangle cueing paradigm (Egly, Driver, \& Rafal, 1994). In this paradigm, participants are presented with two rectangles, one positioned on either side of fixation, followed by a brief illumination of one rectangle's end (cue), then the filling-in of one rectangle's end (target). The target can appear at the valid location, the invalid location within the same rectangle, or the equidistant (from the cue) invalid location within the noncued rectangle. Studies have shown that participants respond faster to valid than to invalid targets. This cue validity effect reflects an orienting of attention to the locus of the cue. More critically, however, studies have also shown that participants respond faster to targets appearing at the invalid same-object location than to those at the invalid different-object location. This same-object advantage reflects increased object-based attentional priority to, and selection of, dimensions of the cued object. Interestingly, the same-object advantage that emerges from this paradigm (and others) is usually small and susceptible to variation, whereas space-based effects are usually large and robust (for a review, see Reppa, Schmidt, \& Leek, 2012). Indeed, numerous studies have not shown a significant 
same-object advantage (e.g., Law \& Abrams, 2002; Shomstein \& Behrmann, 2008), or have found reversals of those effects (a same-object cost; Chen \& Huang, 2015; see, e.g., Pilz, Roggeveen, Creighton, Bennett, \& Sekuler, 2012). Other researchers, in contrast, have observed a particularly large same-object advantage, which can even be larger than the spatial effect (e.g., Greenberg, Rosen, Cutrone, \& Behrmann, 2015). This inconsistency in the emergence, sign, and magnitude of object-based effects - relative to spacebased effects - has raised questions about the boundary conditions of object-based selection.

The variations in object-based effects could be due to a number of factors. One intriguing possibility concerns differences in target-object integration across commonly used paradigms. In the aforementioned double-rectangle paradigm, for example, participants detect or identify targets that appear 'on' an object (Egly et al., 1994). In the 'wrenches' paradigm of Watson and Kramer (1999), however, participants identify targets that appear as part 'of' an object. Specifically, in this task, participants are presented with two wrench-like stimuli on either side of fixation. Each wrench could have an end with a bend, an end with a gap, or both. Participants are asked to judge whether one or two of these target properties are present in the object display. Researchers typically find that responses are faster when both properties are part of the same wrench rather than on different wrenches. This result, much like the same-object advantage found in the double-rectangle paradigm, is taken as evidence of object-based selection. Interestingly, though, the same-object advantage reported in the wrenches paradigm is larger (66 ms; Watson \& Kramer, 1999) than the same-object advantage reported in the doublerectangle paradigm (13 ms; Egly et al., 1994). Some researchers have attributed this difference in the magnitude of the same-object advantage across paradigms to dissimilarities in target-object integration (e.g., Pilz et al., 2012). The assertion is that the appearance of the target after, and distinct from, the objects in the double-rectangle paradigm may not encourage participants to perceive (at least not on all trials) the target as an essential component of the objects (e.g., Richard, Lee, \& Vecera, 2008). This weak target-object integration could explain the small(er) same-object advantage in the doublerectangle paradigm because participants need not adopt a strategy whereby they attend strictly to the objects to complete the task efficiently. In contrast, the appearance of the target as part 'of' objects in the wrenches paradigm encourages participants to perceive, on all trials, the targets as essential parts of the objects. This strong target-object integration could explain the large(r) same-object advantage in the wrenches paradigm because participants need to adopt a strategy whereby they select all object parts to complete the task efficiently. The robustness of object selection may, therefore, be modulated by the strength of target-object integration, insofar as this factor affects the strategy that participants use when deciding which objects should be selected. To our knowledge, the object-based effects yielded by the double-rectangle and wrenches paradigms have not been contrasted in the same participants, and thus this explanation of the incongruous magnitudes of object-based selection is not yet confirmed.

Another possible explanation for variations in object-based effects, which is not mutually exclusive from the first, concerns differences in the distributions of attention across paradigms. In particular, attention is focused narrowly prior to target onset in the double-rectangle paradigm because a peripheral cue precedes the target, whereas, in the wrenches paradigm, attention is focused widely prior to target onset because no cue precedes the targets. Lamy and Egeth (2002) investigated whether or not the distribution of attention modulates object-based effects. In their task, participants judged whether two targets appearing simultaneously 'on' objects were the same or different (Exp. 1). These targets were not preceded by a cue, and thus attention was focused widely prior to target onset, as in the wrenches paradigm (Watson \& Kramer, 1999). Interestingly, the researchers found that response latencies did not differ when the targets appeared 'on' the same object as opposed to 'on' different objects; that is, a same-object advantage did not emerge with weak targetobject integration when attention was focused widely. Two accounts have been put forward to explain this finding. First, the pattern of results may indicate that focusing attention narrowly by way of a spatial cue enables participants to shift attention from one possible target location to another location - a strategy, according to Lamy and Egeth, that may be necessary for driving object-based selection when the targets appear 'on' an object (i.e., the attention-shifting account). Second, and alternatively, the pattern of results may indicate that focusing attention narrowly by way of a spatial cue enables participants to prioritize, then select, a cued object-a strategy, according to Drummond and Shomstein (2010), that may be necessary for driving object-based selection when targets appear 'on' an object, at least when no alternative strategy is in place to pinpoint the most likely target location (i.e., the attentional-prioritization account). Regardless of which account accurately explains the results of Experiment 1 , both proposals indicate that focusing attention narrowly may be a prerequisite for object selection to occur when targets and objects are weakly integrated. Lamy and Egeth went on to test this hypothesis by presenting the targets sequentially rather than simultaneously (Exp. 3). The researchers reasoned that this design would encourage participants on invalid trials to focus attention narrowly on the Target 1 locus, and then to shift it to the Target 2 locus. Indeed a same-object advantage emerged under such conditions, thus suggesting that the occurrence of object-based selection (during weak target-object integration) relies on an initial narrowing of attention by way of a spatial cue. It is still an open question, however, as to the manner by which such selection would be affected, if at all, by 
the distribution of attention when the target and object are integrated strongly (i.e., when the targets are changes to, or part 'of', an object).

A third possible modulatory factor of object-based selection is object orientation. Previous studies have suggested that object orientation can affect the sign of object-based selection effects (Conci \& Müller, 2009; de-Wit, Kentridge, \& Milner, 2009; Harrison \& Feldman, 2009; Pilz et al., 2012). Pilz et al., for example, asked participants to identify a target in the double-rectangle cueing paradigm (Exp. 1) that was preceded by an $80 \%$-valid cue (cf. Moore, Yantis, \& Vaughan, 1998). The researchers found evidence of object-based selection, but, importantly, the sign of that effect was modulated by object orientation. In particular, a same-object advantage emerged when objects were oriented horizontally and a same-object cost (i.e., faster responses on invalid different-object than on invalid same-object trials) emerged when objects were oriented vertically. The authors suggested that the modulation of object selection by object orientation could be due to the ease with which object-based attention is allocated when crossing the vertical as opposed to the horizontal meridian (see also deWit et al., 2009), which primarily happens when attention is shifted endogenously as opposed to exogenously. This suggestion is based on findings that have shown modulations of spatial selection by meridians when attention is oriented endogenously (e.g., Botta, Santangelo, Raffone, Lupiáñez, \& Belardinelli 2010). Indeed, Greenberg et al. (2014) showed that the orientation effects observed in the Pilz et al. study disappear when controlling for shifts across the meridians. The researchers compared object-based selection along the vertical meridian (by subtracting same-object trials in the vertical condition from different-object trials in the horizontal condition) to selection along the horizontal meridian (by subtracting same-object trials in the horizontal condition from different-object trials in the vertical condition). This analysis revealed object-based selection along the vertical meridian, when parceling out shifts across the vertical meridian, which is not significantly different from object-based selection along the horizontal meridian, when parceling out shifts across the horizontal meridian. Hence, although it has previously been shown that the mode of orienting (exogenous vs. endogenous) may modulate the emergence or magnitude of object-based selection (e.g., Abrams \& Law, 2000; Macquistan, 1997), it is also possible that this factor changes the propensity for object orientation to vary the sign of this selection.

Despite considerable research investigating the manner in which object-based effects manifest, relatively little work has investigated the reasons for which object-based selection is less robust than spatial selection. With this gap in mind, the aim of the present study was to systematically explore the manner by which target-object integration, attention distribution, and object orientation modulate object-based selection effects. To pursue this aim, we used modified versions of the double-rectangle paradigm (Egly et al., 1994) and the wrenches paradigm (Watson \& Kramer, 1999). In Experiment 1, we sought to investigate the manner by which target-object integration and object orientation may modulate object selection when attention is initially focused widely. Specifically, we manipulated target-object integration within subjects, across sessions. On one session, participants judged whether two targets that appeared 'on' objects were the same or different. On the subsequent session, they judged whether two targets that were changes 'of' objects were the same or different. The objects were oriented either horizontally or vertically across blocks. Attention was focused widely in this experiment because the targets were not preceded by a cue. In Experiment 2, we modified the design so that an informative $(75 \%$-valid) cue preceded a sole target that participants identified. This modification allowed us to investigate the manner by which target-object integration and object orientation may modulate object-based selection when attention is initially focused narrowly. Finally, in Experiment 3, we encouraged participants to shift spatial attention purely exogenously by rendering the spatial cue uninformative ( $33 \%$ valid) of target location. This design enabled us to investigate whether or not the effect of object orientation on object-based selection is a result of the manner by which attention is oriented (endogenously vs. exogenously), as has been postulated by researchers (e.g., Pilz et al., 2012).

\section{Experiment 1}

The aim of Experiment 1 was to investigate the contribution of target-object integration to object-based selection when attention is focused widely prior to target onset, as in the wrenches paradigm (Watson \& Kramer, 1999), rather than narrowly prior to target onset, as in the double-rectangle cueing paradigm (Egly et al., 1994). We also sought to investigate the contribution of object orientation. The manipulation of target-object integration was conducted across sessions, whereas the manipulation of object orientation was conducted across blocks. Participants were presented with two surfboardshaped objects with a circle at each end (see Fig. 1) on both days. In one session, two numbers appeared on two (of the four possible) surfboard ends. The numbers were either odd or even, and participants were asked to indicate whether the numbers were of same or different parities. We categorized the target-object relationship here as being 'on' the objects, given that the targets were rendered on top of the objects. In another session, two (of the four possible) circles extended out toward the center of the object (see Fig. 1). These produced "notches" that could be either narrow or wide, and participants were asked to indicate whether the two notches were of same or different thicknesses. We categorized the targetobject relationship here as being 'of' the objects, given that the 
a)

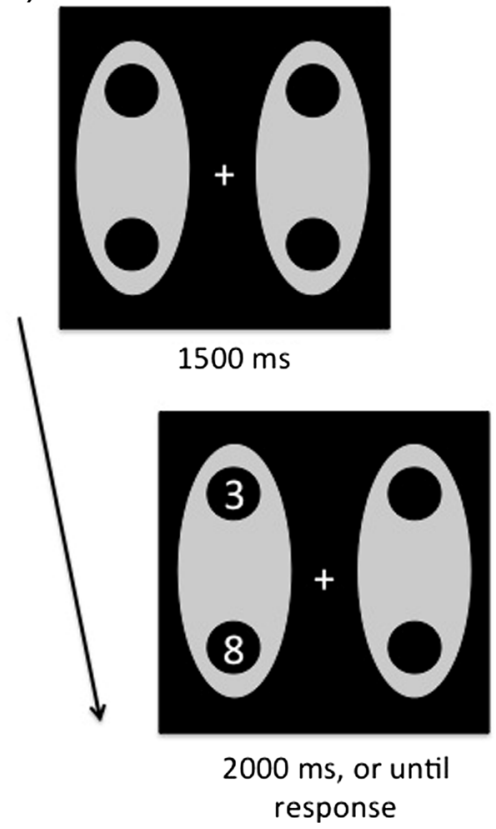

b)
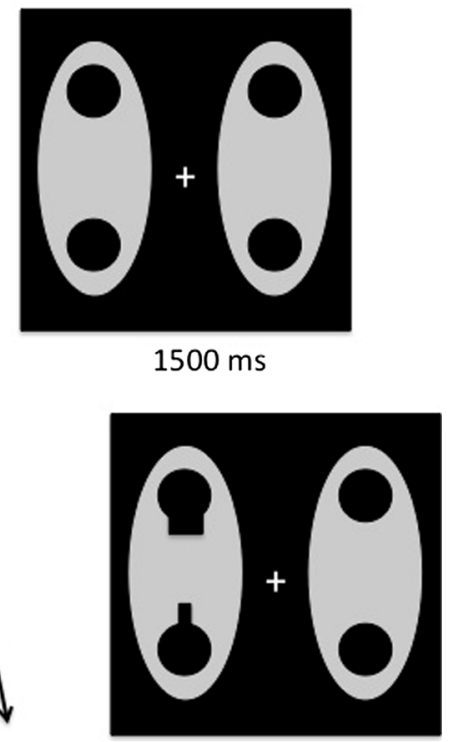

2000 ms, or until response

Fig. 1 Task procedure for Experiment 1 when (a) targets are 'on' an object versus (b) 'of' an object. A 'same-object' trial is depicted, given that targets appear within the same object, although they are dissimilar in either (a) parity or (b) thickness. The objects in this figure are oriented vertically

targets involved a structural change of object shape. On both days, we predicted that object-based selection would emerge as better performance on target judgments when the stimuli appeared within the same object rather than within different objects (i.e., a same-object advantage). If the magnitudes of the same-object advantage differed across target-object integration strengths, and also across object orientations, then this result would show that both factors modulate object-based selection when attention is initially distributed.

\section{Method}

Participants Twenty-four participants (18 females, six males; mean $+S D$ age, $28+10$ years) at the University of Wisconsin-Milwaukee (UWM) participated in the study for $\$ 20$ or course credit. The UWM Institutional Review Board approved the study.

Stimuli The surfboard-shaped objects comprised gray ovals that measured $13^{\circ} \times 2^{\circ}$ of visual angle in the horizontal condition, and $2^{\circ} \times 13^{\circ}$ of visual angle in the vertical condition. The objects were positioned $9^{\circ}$ apart, and a white fixation cross (20-point Monaco font) was centered between them. Each object contained black circles of size $1^{\circ} \times 1^{\circ}$ that were positioned $0.5^{\circ}$ from its ends. The target notches were wide $\left(0.4^{\circ} \times 0.6^{\circ}\right.$ when the objects were oriented horizontally, and $0.6^{\circ} \times 0.4^{\circ}$ when the objects were oriented vertically) or narrow $\left(0.4^{\circ} \times 0.4^{\circ}\right.$ when the objects were oriented horizontally, and $0.3^{\circ} \times 0.4^{\circ}$ when the objects were oriented vertically) black rectangles that extended from the midpoint of the circle. The size of the narrow targets was determined using a staircase procedure in a pilot experiment. ${ }^{1}$ The targets were the numerical characters 3 and 8 (in 20-point Monaco font), which appeared centered within the circle, rendered in white. All stimuli were presented on a black background using a 17in. CRT monitor at a screen resolution of $1,024 \times 768$ and a vertical refresh rate of $100 \mathrm{~Hz}$. We supported participants' heads using a chinrest at a viewing distance of $50 \mathrm{~cm}$.

Procedure The within-subjects factors manipulated in this experiment were Target-Object Integration ('on' object, 'of' object), Object Orientation (horizontal, vertical), and Target Location (same object, different object). Target-object integration was varied randomly within subjects, across consecutive sessions. Specifically, on Session 1, half of the participants were presented with targets that appeared 'on' objects, and the other half were presented with targets that appeared as

\footnotetext{
${ }^{1}$ We used a QUEST adaptive staircase procedure (Watson \& Pelli, 1983) in a pilot experiment $(n=7)$ to evaluate the minimum difference in thickness required for participants to correctly discriminate between the targets on $87 \%$ of trials. In each block (one for horizontal and one for vertical), two QUEST blocks ran concurrently and were interleaved. Specifically, we varied the thickness of the narrow target from trial to trial separately for each target location condition. Each QUEST block contained 40 trials. Participants were asked to determine whether the targets were of the same or different thicknesses. The measured thresholds differed across object orientations, $F(6)=7.33, p<.05$, such that the threshold was larger for vertical objects $(M=8, S D=1.90)$ than for horizontal objects $(M=6, S D=0.94)$.
} 
part 'of' objects. Participants were presented with the complementary target on Session 2. Participants completed 20 practice trials and 768 experimental trials on each day. Thus, there were 192 trials in each of the four conditions for each targetobject integration condition. The trial structure was as follows (Fig. 1): A central fixation cross was presented along with two surfboard-shaped objects oriented horizontally on half of the blocks and vertically on the other half. Block order was randomized. After 1,500 ms, the targets appeared within either the same object or different objects. The targets appearing within the same object or within different objects were always equidistant. During the target 'on' condition, participants indicated whether the targets were of the same or different parities. During the target 'of' condition, participants indicated whether the notches were of same or different thicknesses. We asked participants to make their response as quickly and accurately as possible by pressing one of two horizontally aligned buttons with the corresponding index finger. The mapping between target and response was counterbalanced. The targets remained on the screen for 2,000 ms or until a response was detected. All trials were randomized. The next trial began following an intertrial interval of 400,600 , or $800 \mathrm{~ms}$.

\section{Results}

Response latencies The dependent variable was the mean reaction times for correct responses $(M=90 \%, S D=9 \%)$. Response latencies less than $100 \mathrm{~ms}(0.04 \%)$ or greater than $1,500 \mathrm{~ms}(3.11 \%)$ were labelled as anticipations and misses, respectively, and discarded. A $2 \times 2 \times 2$ repeated measures analysis of variance (ANOVA) was conducted on response latencies, with the factors Target-Object Integration ('on' object, 'of' object), Object Orientation (horizontal, vertical), and Target Location (same object, different object). We found a main effect of object orientation, $F(1,23)=30.33, p<.0001$, such that participants' response latencies were shorter when the objects were vertical $(M=898 \mathrm{~ms}, S D=114)$ than when they were horizontal $(M=929 \mathrm{~ms}, S D=116)$. The analysis also revealed a main effect of target location, $F(1,23)=11.07$, $p<.001$, indicating shorter response latencies when targets appeared within different objects $(M=910 \mathrm{~ms}, S D=119)$ rather than the same object $(M=917 \mathrm{~ms}, S D=113)$. This main effect indicates an overall same-object cost rather than a same-object advantage. The main effects, however, were qualified by several two-way interactions: Target-Object Integration $\times$ Object Orientation, $F(1,23)=14.94, p<$ .0001; Target-Object Integration $\times$ Location, $F(1,23)=$ $18.33, p<.0001$; and Object Orientation $\times$ Location, $F(1$, $23)=166.19, p<.0001$. We found no other significant effects (all $F_{\mathrm{S}}<2.98$; see Table 1). The interactions are each detailed below.

Target-object integration and object orientation This interaction was driven by a difference in response latencies to targets when they appeared as changes 'of' an object as a function of object orientation (Fig. 2). Specifically, when targets were changes 'of' an object, we found that participants' response latencies were shorter when the objects were oriented vertically

Table 1 Mean response latencies and standard deviations as a function of target-object integration, attention distribution, and object orientation for Experiments 1-3

\begin{tabular}{|c|c|c|c|c|c|c|c|c|}
\hline & \multirow[b]{4}{*}{ Target-Object Integration } & & \multicolumn{6}{|c|}{ Object Orientation } \\
\hline & & & \multicolumn{3}{|c|}{ Horizontal } & \multicolumn{3}{|c|}{ Vertical } \\
\hline & & & \multicolumn{3}{|c|}{ Cue-Target Location } & \multicolumn{3}{|c|}{ Cue-Target Location } \\
\hline & & & Valid & Same Object & Different Object & Valid & Same Object & Different Object \\
\hline \multirow[t]{4}{*}{ Experiment 1 (No Cue) } & \multirow[t]{2}{*}{ Target "on" object } & $M$ & NA & 887 & 939 & NA & 931 & 888 \\
\hline & & $S D$ & & 102 & 104 & & 109 & 110 \\
\hline & \multirow[t]{2}{*}{ Target “of” object } & $M$ & NA & 935 & 953 & NA & 914 & 858 \\
\hline & & $S D$ & & 124 & 127 & & 116 & 114 \\
\hline \multirow[t]{4}{*}{ Experiment 2 (Informative Cue) } & \multirow[t]{2}{*}{ Target "on" object } & $M$ & 629 & 736 & 781 & 630 & 775 & 745 \\
\hline & & $S D$ & 119 & 95 & 102 & 115 & 100 & 106 \\
\hline & \multirow[t]{2}{*}{ Target “of” object } & $M$ & 608 & 743 & 803 & 596 & 769 & 735 \\
\hline & & $S D$ & 83 & 109 & 143 & 86 & 149 & 128 \\
\hline \multirow[t]{4}{*}{ Experiment 3 (Uninformative Cue) } & \multirow[t]{2}{*}{ Target "on" object } & $M$ & 652 & 703 & 717 & 626 & 684 & 682 \\
\hline & & $S D$ & 103 & 97 & 91 & 85 & 82 & 83 \\
\hline & \multirow[t]{2}{*}{ Target "of" object } & $M$ & 644 & 722 & 768 & 631 & 725 & 716 \\
\hline & & $S D$ & 56 & 713 & 116 & 61 & 101 & 94 \\
\hline
\end{tabular}




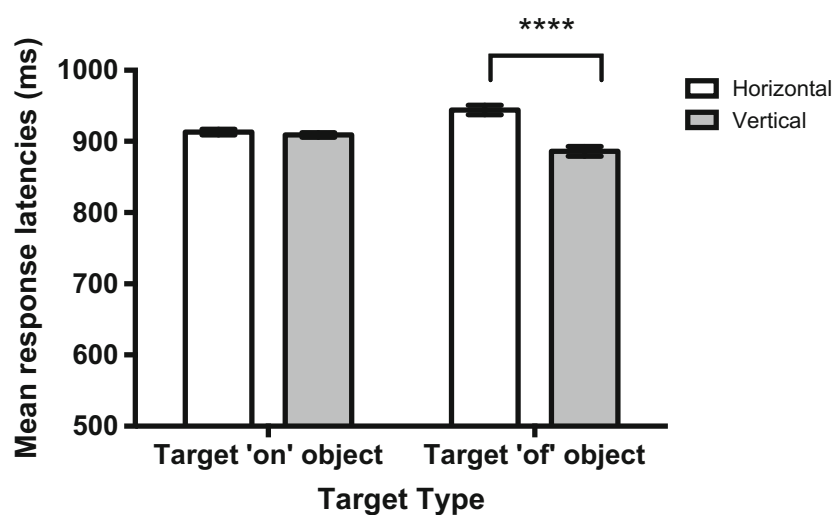

Fig. 2 Mean response latencies when targets appear embedded 'on' an object versus 'of' an object, as a function of object orientation, in Experiment 1. Error bars represent the within-subjects standard errors of the means. ${ }^{* * * *} p<.0001$

$(M=886 \mathrm{~ms}, S D=118)$ versus horizontally $(M=944 \mathrm{~ms}, S D=$ $125), t(23)=4.92, p<.0001$. In contrast, when targets appeared 'on' an object, we found that participants' response latencies were no different when the objects were oriented vertically ( $M$ $=909 \mathrm{~ms}, S D=111)$ versus horizontally $(M=913 \mathrm{~ms}, S D=$ $105), t(23)=0.84, p=.41$. Thus, participants made faster judgments when the targets appeared as part 'of' vertical rather than horizontal objects.

Target-object integration and target location We calculated difference scores for the same-object advantage (i.e., differentobject location response latencies minus same-object location response latencies), separately for each level of target-object integration, to examine this interaction. We then conducted planned paired comparisons across those scores. The interaction was driven by a difference in the magnitudes of object-based selection between target-object integration conditions (see

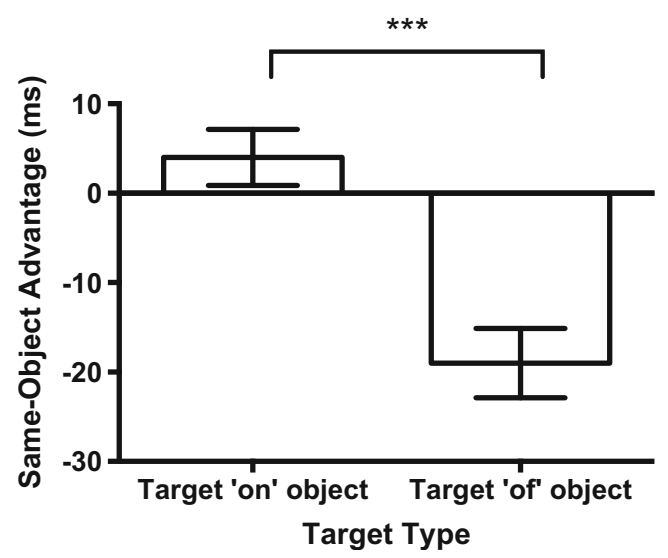

Fig. 3 Object-based effects in Experiment 1 when the targets appear either "on" or "of" the same versus different objects. The effects were calculated by subtracting response latencies on same-object trials from different-object trials. Therefore, positive values indicate a same-object advantage and negative values indicate a same-object cost. Error bars represent the within-subjects standard errors of the mean. ${ }^{* * *} p<.001$
Fig. 3). Specifically, object-based selection was stronger when the targets were changes 'of' objects $(M=-19 \mathrm{~ms}$, $S D=19)$ rather than when targets appeared 'on' an object $(M=4 \mathrm{~ms}, S D=15), t(23)=4.28, p<.001$. Actually, we found no evidence of object-based selection for targets 'on' an object, $t(23)=1.44, p=.16$. Notably, however, the sign of the object-based selection effects for targets that were changes 'of' an object ran contrary to our hypothesis, reflecting a same-object cost rather than an advantage.

Orientation and target location Parallel to the analysis above, we calculated difference scores for the same-object advantage, separately for each object orientation, then conducted planned paired comparisons across those scores. The signs of the object-based selection effects were significantly different when the objects were oriented horizontally $(M=35$ $\mathrm{ms}, S D=20)$ versus vertically $(M=-49 \mathrm{~ms}, S D=18), t(23)=$ $12.89, p<.0001$. This result indicates that a same-object advantage is limited to conditions when objects are horizontal, and a same-object cost is limited to conditions when objects are vertical (see Fig. 4).

Meridian control analysis Importantly, Pilz et al. (2012) had also observed this same-object cost for the vertical orientation. There are three possible explanations for this finding. The first explanation posits that shifts of object-based attention across the vertical meridian (i.e., along the horizontal meridian) are facilitated relative to shifts across the horizontal meridian (i.e., along the vertical meridian). Hence, shifts of object-based attention between vertical objects are speeded as compared to shifts within a vertical object. By contrast, the second explanation posits that shifts of object-based attention across the horizontal meridian are inhibited relative to shifts across the vertical meridian. Hence, under this latter explanation, shifts of object-based attention within vertical objects are inhibited relative to shifts between vertical

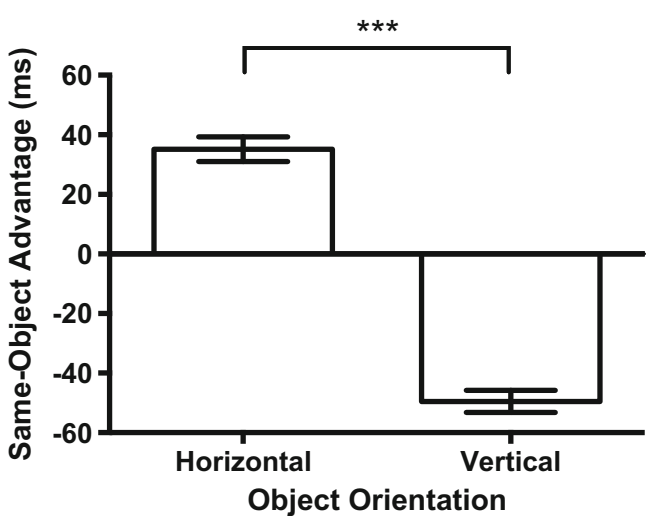

Fig. 4 Object-based effects in Experiment 1 when the objects appeared either horizontally or vertically. Positive values indicate a same-object advantage, and negative values indicate a same-object cost. Error bars represent the within-subjects standard errors of the mean. ${ }^{* * *} p<.001$ 
objects. The third explanation allows for the possibility that the same-object cost that we observed is driven by a combination of these factors. Independent of which explanation is correct, however, all accounts indicate that shifts of object-based attention are more efficient when crossing the vertical, relative to the horizontal, meridian.

Thus, following Greenberg and colleagues (2014), we sought to parcel out shifts of attention across the vertical meridian in order to investigate the allocation of object-based attention along the vertical meridian. Specifically, we deducted the response latencies on same-object trials in the vertical condition from those on different-object trials in the horizontal condition (i.e., a meridian control difference score; see Fig. 5). These conditions were selected because they both involve shifts of object-based attention that always cross the horizontal meridian (and never cross the vertical meridian). Thus, if we were to find that response latencies in these two conditions were not significantly different from each other, this result would indicate no evidence of object-based selection along the vertical meridian. If, however, we were to find that response latencies were slower in the different-object condition than in the same-object condition, this result would be indicative of object-based selection along the vertical meridian - that is, that an advantage is afforded to shifts of objectbased attention occurring across the horizontal meridian within the same object as opposed to shifts of object-based attention occurring across the horizontal meridian between objects. In planned comparisons, we conducted a one-sample $t$ test on the meridian control difference scores to examine whether or not the result was significantly different from zero. The analysis revealed that participants' response latencies to the targets were $24 \mathrm{~ms}$ shorter when they appeared within the same object in the vertical orientation rather than within different objects in the horizontal orientation, $t(23)=4.12, p<.001$. This result shows, despite the orientation differences that we observed, that object-based selection occurred along the vertical meridian when attention was initially focused widely, and that the direction of that selection was positive (Fig. 6).

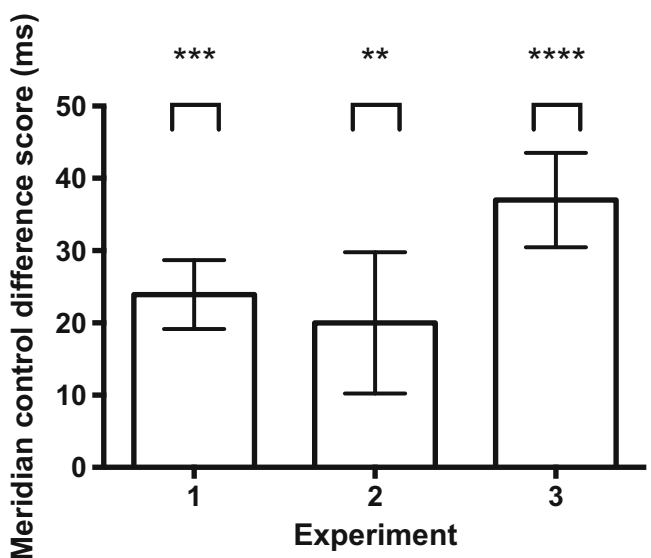

Fig. 6 Shown are object-based selection effects along the vertical meridian for Experiments 1, 2, and 3, respectively. Error bars represent the within-subjects standard errors of the mean. ${ }^{* *} p<.01,{ }^{* * *} p<.001$, ${ }^{* * * *} p<.0001$

\section{Discussion}

In Experiment 1, we investigated the contributions of targetobject integration and object orientation to object-based selection when attention is focused widely prior to target onset. This investigation yielded three important sets of results: (a) Target-object integration affects the emergence of objectbased selection. Specifically, we found evidence of objectbased selection when the targets were changes 'of' objects, but not when they appeared 'on' objects. This difference in the emergence of object-based selection between target-object integration conditions is consistent with the finding that targets appearing 'on' objects produce object-based effects only when attention is initially focused narrowly (Lamy \& Egeth, 2002). Contrary to the predictions, however, the object-based selection we observed for targets appearing as changes 'of' an object represented an instance of a same-object cost rather than an advantage (for similar findings in divided-attention tasks, see also Behrmann, Zemel, \& Mozer, 1998; Cepeda \& Kramer, 1999; Chen \& Huang, 2015; Davis \& Holmes, 2005;

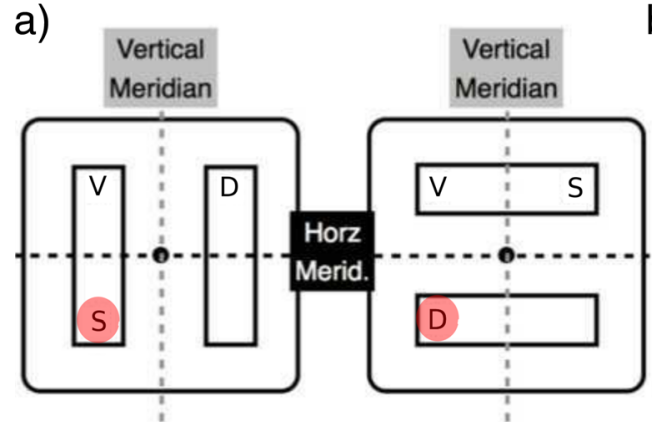

Fig. 5 Graphic of the calculations used to control for attention shifts along the vertical meridian; specifically, we subtracted response times on same-object trials in the vertical condition from those in differentobject trials in the horizontal condition. This calculation was done b)

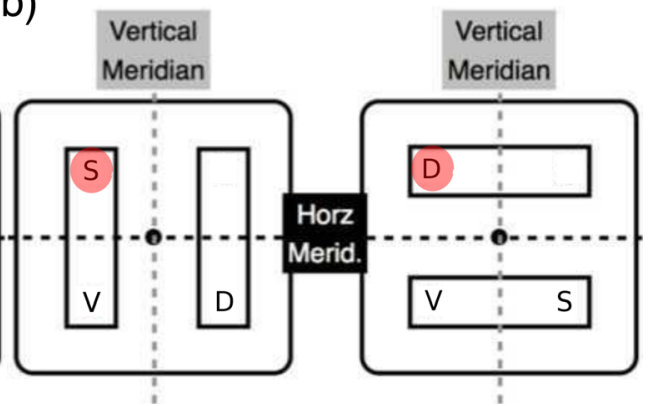

independent of target location. We have depicted here calculations for trials in which the target appeared in (a) the lower left screen location and (b) the upper left screen location 
Davis, Welch, Holmes, \& Shepherd, 2001; Lavie \& Driver, 1996). This same-object cost is common to divided-attention tasks in which participants are asked to judge between two highly similar or identical targets (Chen \& Huang, 2015) and reflects greater binding of features from distinct, albeit neighboring, objects than for features from the same object (Davis \& Holmes, 2005). Indeed, it is possible that participants are motivated to attend strictly to the objects when targets and objects are strongly integrated (i.e., when the targets are part 'of' the objects), and thus, when completing the same-different task, they are prone to adopting a strategy whereby they compare whole objects rather than distinct features of those objects (Neill, Li, Seror, \& O'Connor, 2009). Consequently, when the targets appear within different objects, participants may base their response on whether or not the two objects match. This strategy may, therefore, lead to a facilitation of response latencies on those trials. In contrast, when the targets appear within the same object, participants are unable to base their response on whether or not two objects match, because such a strategy would be uninformative as to whether the targets are themselves the same or different. This strategy may, therefore, hinder response latencies on those trials. (b) Object orientation affects the sign of object-based selection effects. In particular, we found a same-object advantage when objects were horizontal, and a same-object cost when objects were vertical. This pattern of results is consistent with that of Pilz et al. (2012). (c) Nevertheless, we did find evidence of object-based selection along the vertical meridian. Importantly, this result indicates that the same-object cost emerging in the vertical orientation is not a reflection of a lack of object-based selection of vertical objects per se. Instead, our analysis shows that this same-object cost appears because the efficiency of attention allocation is affected when crossing the visual-field meridians (for a more detailed exploration of this issue, see Barnas \& Greenberg, 2016). Given that these findings emerged under conditions of wide attention allocation prior to target onset, it is pertinent to ask: What advantage is afforded by focusing attention narrowly prior to target onset? We investigated this question in Experiment 2 by introducing a peripheral spatial cue that was informative of the target location. The purpose of this cue was to encourage participants to initially focus attention narrowly on each trial.

\section{Experiment 2}

In Experiment 2 we ascertained the contributions of targetobject integration and object orientation to object-based selection when attention is initially focused narrowly, as in the double-rectangle cueing paradigm (Egly et al., 1994). Participants were again presented with two surfboard-shaped objects. On one session, a target digit was presented, which could either be odd or even. Participants indicated the parity of this sole target. On another session, the target was a notch that was a part of one surfboard, which could be either narrow or wide. Participants indicated the thickness of this sole notch. Hence, as in Experiment 1, we manipulated target-object integration within subjects, across sessions. Object orientation was manipulated across blocks. Importantly, we introduced a peripheral, $75 \%$-valid spatial cue. The cue, which preceded target presentation, served to encourage participants to focus attention narrowly at a possible target location. Thus, we expected space-based effects to emerge as better performance on target judgments when the stimulus appeared within the location indicated by the cue, as opposed to the invalid sameobject location. In contrast, we expected object-based selection to emerge as better performance on target judgments when the stimulus appeared within the invalid location of the same object rather than within the invalid, yet equidistant, location on the different object (i.e., a same-object advantage). If we were to find that both target-object integration conditions ('on' vs. 'of') produced a same-object advantage that varied in magnitude and also across object orientations, this result would suggest that both factors modulate object selection when attention is initially focused narrowly.

\section{Method}

Participants Twenty-four participants (21 females, three males; mean $+S D$ age, $22+7$ years) at UWM participated in the study for $\$ 20$ or course credit. The UWM Institutional Review Board approved the study.

Stimuli The stimuli were the same as those used in Experiment 1, with the addition of a yellow rectangular bar cue that measured $1^{\circ} \times 2^{\circ}$ in the horizontal condition and $2^{\circ} \times$ $1^{\circ}$ in the vertical condition. The bar cue appeared $0.4^{\circ}$ to the left or right of the objects in the horizontal condition, and $0.4^{\circ}$ above or below the objects in the vertical condition. Given that participants were no longer making a judgment between targets, we equated the sizes of narrow 'of' object targets, such that they were $0.4^{\circ} \times 0.4^{\circ}$ in both orientations.

Procedure The within-subjects factors manipulated in this experiment were Target-Object Integration ('on' object, 'of' object), Object Orientation (horizontal, vertical), and CueTarget Validity (valid, invalid same-object, invalid differentobject). Target-object integration was varied randomly within subjects, across consecutive sessions. Participants completed 24 practice trials and 768 experimental trials on each day. Thus, they performed 192 trials in each of the four conditions for each target-object integration condition. The trial structure was as follows (Fig. 7): A central fixation was presented along with two surfboard-shaped objects for 1,000 ms. The objects were oriented horizontally on half of the blocks and vertically on the other half. Block order was randomized. An 
a)

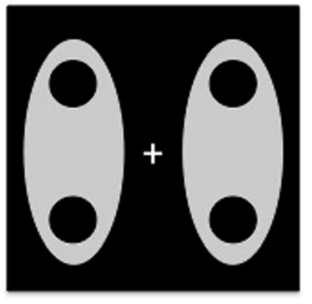

$1000 \mathrm{~ms}$

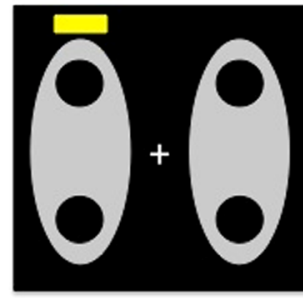

$100 \mathrm{~ms}$

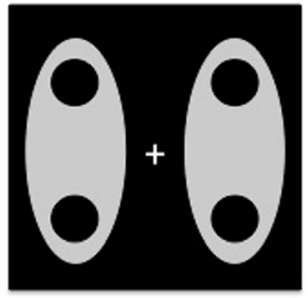

$400 \mathrm{~ms}$

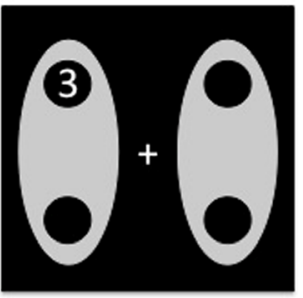

2000 ms, or until response

b)

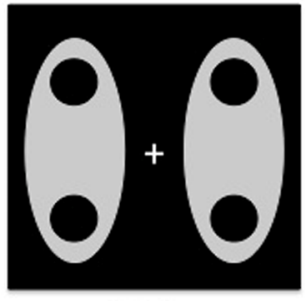

$1000 \mathrm{~ms}$

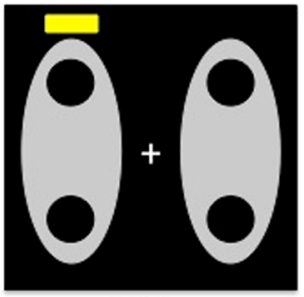

$100 \mathrm{~ms}$

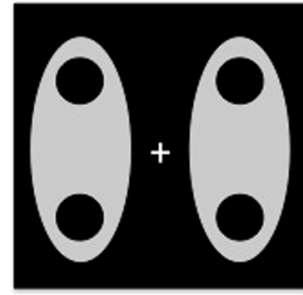

$400 \mathrm{~ms}$

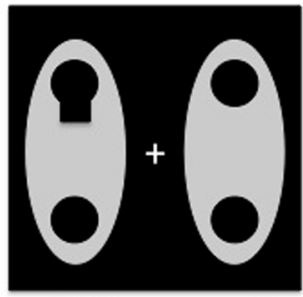

$2000 \mathrm{~ms}$, or until response

Fig. 7 Task procedure for Experiment 2 when targets were (a) 'on' an object versus (b) 'of' an object. A 'valid' trial is depicted, given that the target appears within the same location and object denoted by the cue

informative cue (75\% valid) was then presented for $100 \mathrm{~ms}$. The cue was followed by an interstimulus interval of $400 \mathrm{~ms}$, at which point the target appeared. The targets could be presented in the same location denoted by the cue (valid trial), at a different location than the one denoted by the cue but on the same object (invalid same-object trial), or at a different location and on a different object than the one denoted by the cue (invalid different-object trial). The targets appearing in the invalid locations were equidistant from the valid location. Participants indicated whether the target was odd or even when completing the 'on' object version of the task, or whether the thickness of the notches was narrow or wide when completing the 'of' object version of the task. All other task parameters, including the overall trial length, were the same as in Experiment 1.

\section{Results}

Response latencies The dependent variable was the mean response latencies for correct responses $(M=89 \%, S D=$ $8 \%)$. Response latencies less than $100 \mathrm{~ms}(0.03 \%)$ or greater than $1,500 \mathrm{~ms}(1.09 \%)$ were labelled as anticipations and misses, respectively, and discarded. A $2 \times 2 \times 3$ repeated measures ANOVA was conducted on response latencies, with the factors Target-Object Integration ('on' object, 'of' object), Object Orientation (horizontal, vertical), and Cue-Target Validity (valid, invalid same-object, invalid different-object).
We found a significant main effect of object orientation, $F(1$, $23)=4.75, p<.05$, such that participants were faster to identify the target when it appeared within a vertical object $(M=$ $708 \mathrm{~ms}, S D=133)$ rather than a horizontal object $(M=717$ $\mathrm{ms}, S D=131)$. Importantly, we found a significant main effect of validity, $F(2,46)=65.09, p<.0001$. Planned paired comparisons revealed that participants' response latencies to the target were significantly shorter when it appeared in the valid location $(M=616 \mathrm{~ms}, S D=101)$ versus the invalid sameobject location $(M=756, S D=114), t(23)=8.10, p<.0001$, indicative of space-based attentional orienting to the cued location. Planned paired comparisons also revealed that participants' response latencies to the target were significantly shorter when it appeared in an invalid same-object location versus an invalid different-object location $(M=766 \mathrm{~ms}, S D=$ $122), t(23)=2.19, p<.05$, indicative of a same-object advantage. The main effect of validity was, however, qualified by two interactions: one with target-object integration, $F(2,46)=$ $6.25, p<.001$, and one with object orientation, $F(2,46)=$ $20.85, p<.0001$. We, therefore, conducted further paired comparisons to investigate the nature of these interactions. No other significant effects were found (all $F_{\mathrm{S}}<2.43$; see Table 1).

Target-object integration and cue-target validity To investigate this interaction, we calculated difference scores for the cue validity effect (i.e., invalid same-object location minus 
a)

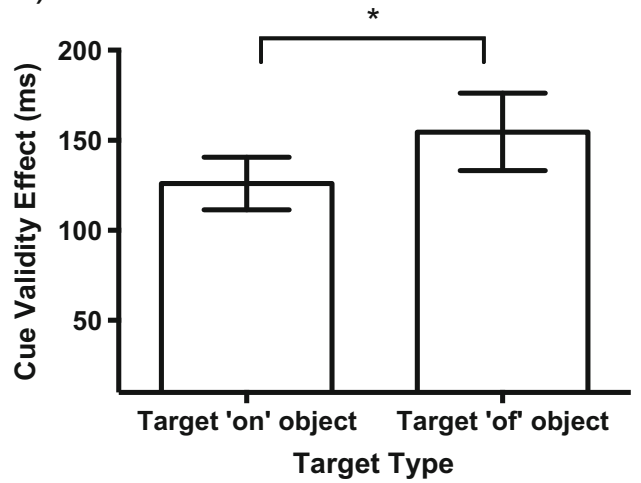

Fig. 8 The (a) space-based and (b) object-based effects (in milliseconds) in Experiment 2, as a function of target-object integration. The spacebased effect was calculated by subtracting the response latencies on valid trials from those on invalid same-object trials; the object-based effect was calculated by subtracting the response latencies on invalid same-object trials from those on invalid different-object trials. Therefore, for space-

valid location) and the same-object advantage, separately for each level of target-object integration. We then conducted planned paired comparisons across those scores. The magnitudes of the space-based effects differed significantly by target-object integration (see Fig. 8). Specifically, the spacebased effect was significantly larger when the targets were changes 'of' the object $(M=155 \mathrm{~ms}, S D=107)$ rather than 'on' the object $(M=126 \mathrm{~ms}, S D=107), t(23)=2.35, p<.05$. We did not find, however, that the magnitudes of object-based selection differed significantly by target-object integration, $t(23)=0.61, p=.55$.

Orientation and cue-target validity As above, we first calculated difference scores for the cue validity effects and the same-object advantages, separately for each object orientation. We then conducted planned paired comparisons across those scores for each attentional effect. The magnitudes of the space-based effect and the signs of the object-based effect

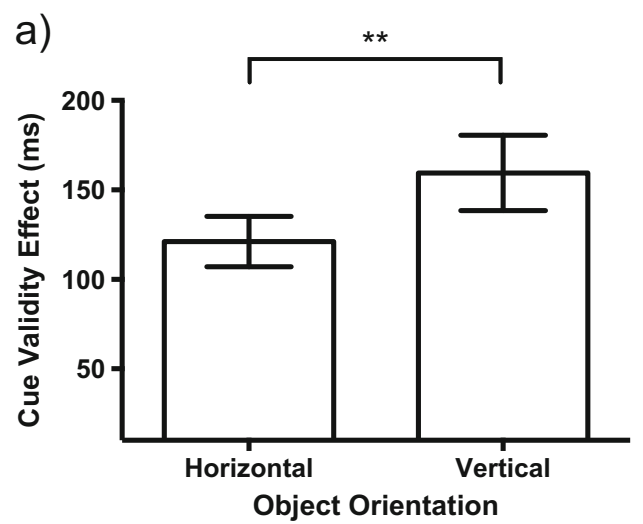

Fig. 9 The (a) space-based and (b) object-based effects in Experiment 2, as a function of object orientation. For space-based selection, positive values indicate a cue validity effect, and negative values would indicate a negative cue validity effect. Similarly, for object-based selection, b)

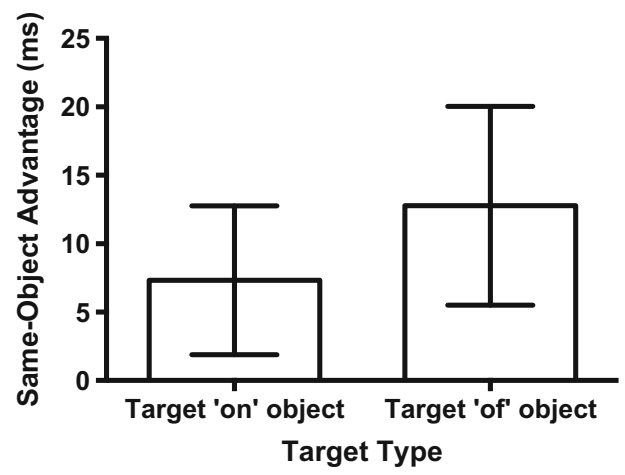

based selection, positive values indicate a cue validity effect, and negative values would indicate a negative cue validity effect. Similarly, for objectbased selection, positive values indicate a same-object advantage, and negative values would indicate a same-object cost. Error bars represent the within-subjects standard errors of the mean. ${ }^{*} p<.05$

differed significantly between orientations (see Fig. 9). Specifically, the space-based effect was significantly larger when the objects were oriented vertically $(M=159 \mathrm{~ms}, S D$ $=101)$ versus horizontally $(M=121 \mathrm{~ms}, S D=113), t(23)=$ $4.24, p<.001$. Moreover, we found that the sign of the objectbased selection effect was significantly different when the objects were horizontal $(M=53 \mathrm{~ms}, S D=112)$ versus vertical $(M=-33 \mathrm{~ms}, S D=121), t(23)=4.77, p<.0001$.

Meridian control analysis Given that we again observed a same-object cost during the vertical condition, as in Experiment 1, we sought to understand the manner in which object-based attention is allocated along the vertical meridian. The analysis revealed that participants' response latencies were $20 \mathrm{~ms}$ shorter when the target appeared at an invalid same-object location in the vertical orientation than when it appeared at a different-object location in the horizontal orientation, $t(23)=3.25, p<.01$ (Fig. 6). This finding indicates that,

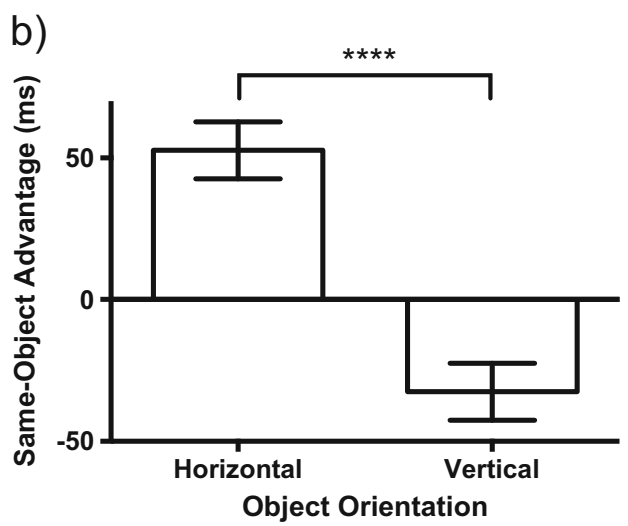

positive values indicate a same-object advantage, and negative values indicate a same-object cost. Error bars represent the within-subjects standard errors of the mean. ${ }^{* *} p<.01, * * * * p<.0001$ 
despite the orientation differences we observed, there was evidence of object-based selection along the vertical meridian during conditions of focal attention.

\section{Discussion}

The purpose of Experiment 2 was two-fold. First, we investigated whether or not the initial capture of attention at a focal location provides an added advantage to object-based selection. Second, and not distinct from the first, we investigated whether this added advantage was modulated by target-object integration and/or object orientation. We note three important findings: (a) Target-object integration did not affect the magnitude of object-based selection. It is likely that this finding emerged in Experiment 2 (but not Exp. 1) because the magnitudes of object-based selection for the different target-object integration conditions are more similar when attention is initially focused narrowly versus widely (the absolute difference in the magnitudes of object-based selection for targets 'on' vs. 'of' objects was $23 \mathrm{~ms}$ in Exp. 1, as compared to $11 \mathrm{~ms}$ in Exp. 2). ${ }^{2}$ It is also likely that this result emerged because both target-object integration conditions yielded object-based selection effects of the predicted sign, now that we no longer used a divided-attention task with highly similar targets. (b) Object orientation continued to affect the sign of object-based effects, such that a same-object advantage was found only when objects were horizontal. (c) We again found evidence of object-based selection along the vertical meridian, after parceling out shifts across the vertical meridian.

As we previously mentioned, the same-object cost when objects are oriented vertically has been attributed to more efficient allocation of object-based attention when crossing the vertical as compared to the horizontal meridian (e.g., de-Wit et al., 2009; Pilz et al., 2012). This "meridian effect" is not restricted to object-based attention. Prior studies have shown that spatial attention is also more efficient when crossing the vertical versus the horizontal meridian, although some studies

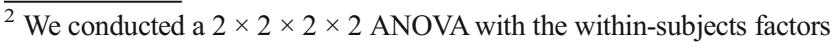
Target-Object Integration ('on' object, 'of' object), Object Orientation (horizontal, vertical), and Validity (same object, different object), and the between-subjects factor Experiment (Exp. 1, Exp. 2). The purpose of this analysis was to investigate whether or not the addition of a spatial cue in Experiment 2 affected the magnitude of object-based effects relative to Experiment 1, in which no spatial cue was utilized. We found that the Experiment factor interacted with Target Type and Validity, $F(1,46)=$ $10.94, p<.01$. We subsequently conducted an independent-samples $t$ test comparing the object-based attention difference scores for each level of target-object integration across experiments. This analysis revealed that the signs of the object-based effect were significantly different in Experiment $1(M=-19 \mathrm{~ms}, S D=19)$ and Experiment $2(M=18 \mathrm{~ms}$, $S D=28$ ) when targets appeared as changes 'of' objects, $t(46)=5.29, p<$ .0001 . In contrast, the signs and magnitudes of the object-based effect when targets appeared 'on' objects were not significantly different in Experiment $1(M=4 \mathrm{~ms}, S D=15)$ and Experiment $2(M=7 \mathrm{~ms}, S D=$ 26), $t(46)=0.39, p=.70$.
}

have shown the reverse effect (e.g., Berlucchi, di Stefano, Marzi, Morelli, \& Tassinari, 1981; Berlucchi, Tassinari, Marzi, \& Di Stefano, 1989; Botta et al., 2010; Downing \& Pinker, 1985; Hughes \& Zimba, 1987; Rizzolatti, Riggio, Dascola, \& Umiltà, 1987; Tassinari, Aglioti, Chelazzi, Marzi, \& Berlucchi, 1987). Indeed, our observation that the space-based effect was larger when objects were oriented vertically rather than horizontally supports the former finding, because it suggests faster reallocation of spatial attention from the cued location to the target location when crossing the vertical, relative to the horizontal, meridian. Importantly, the meridian effect is a known consequence of endogenous, rather than exogenous, shifts of spatial attention (e.g., Botta et al., 2010; Rizzolatti et al., 1987), because only the former requires access to a cognitive representation of visual space (Reuter-Lorenz \& Fendrich, 1992). Given that the participants in our experiments may have shifted attention (at least, partially) endogenously, we sought to encourage exogenous orienting in Experiment 3 by using an uninformative cue. This design allowed us to investigate whether or not the control of attention interacted with the spatial scheme of our environment, and, in turn, affected object-based selection (as it affects spatial selection).

\section{Experiment 3}

In Experiment 3 we examined the hypothesis that the sameobject cost we observed when objects were oriented vertically was due to a (general) advantage in attention allocation efficiency associated with crossing the vertical meridian as compared to crossing the horizontal meridian, which is typically observed only when attention is oriented endogenously (Botta et al., 2010). Hence, we replicated Experiment 2, but now the peripheral cue was uninformative of target location. If the same-object cost found in Experiments 1 and 2 were indeed a result of the meridian effect, which arose because participants shifted attention endogenously (at least partly, in the case of Exp. 2's hybrid cue), then we could make two predictions about what might happen when participants exogenously oriented attention. First, we might find that the allocation of object-based attention was no longer more efficient when crossing the vertical as compared to the horizontal meridian, thus enabling the emergence of a same-object advantage that was not modulated by object orientation. Second, we might find that the allocation of object-based attention was still more efficient when crossing the vertical as compared to the horizontal meridian, but to a lesser extent, thus leading to no object-based effects when objects were oriented vertically. If, however, the same-object cost found in prior experiments was not a result of the meridian effect (or, at least, was not of the same form as the meridian effect in spatial attention-i.e., 
due to endogenous orienting), then we should again find a same-object cost for vertical objects.

\section{Method}

Participants Twenty-four participants (17 females, seven males; mean $+S D$ age, $23+8$ years) at UWM participated in the study for $\$ 20$ or course credit. The UWM Institutional Review Board approved the study.

Stimuli The stimuli were the same as those used in Experiment 2.

Procedure The procedure was also the same as that of Experiment 2, except now the peripheral cue was uninformative of target location ( $33 \%$ valid).

\section{Results}

Response latencies The dependent variable was the mean response latency for correct responses $(M=92 \%, S D=6 \%)$. We discarded responses that were less than $100 \mathrm{~ms}(0.04 \%)$ or greater than $1,500 \mathrm{~ms}(1.38 \%)$ as anticipations and misses, respectively. A $2 \times 2 \times 3$ repeated measures ANOVA was conducted on response latencies, with the factors TargetObject Integration ('on' object, 'of' object), Object Orientation (horizontal, vertical), and Cue-Target Validity (valid, invalid same-object, invalid different-object). We found a significant main effect of object orientation, $F(1,23)$ $=21.14, p<.0001$, such that participants identified the target faster when it appeared within a vertical object $(M=677 \mathrm{~ms}$, $S D=92)$ rather than a horizontal object $(M=701, S D=101)$. The results also revealed a significant main effect of cue-target validity, $F(2,46)=43.68, p<.0001$. Planned paired comparisons showed that participants' response latencies to identify the target were significantly shorter when the target appeared within a valid location $(M=638 \mathrm{~ms}, S D=80)$ rather than within an invalid same-object location $(M=709, S D=$ $91), t(23)=7.13, p<.0001$, indicating space-based attentional orienting to the cued location. Planned paired comparisons also revealed that participants' response latencies to identify the target were significantly shorter when it appeared within the invalid same-object location versus the invalid differentobject location $(M=721 \mathrm{~ms}, S D=100), t(23)=2.62, p<.05$, indicating that a same-object advantage also emerged. The main effect of validity was qualified by two interactions: one with target-object integration, $F(2,46)=10.99, p<.0001$, and one with object orientation, $F(2,46)=10.15, p<.0001$. We also found a significant three-way interaction, $F(2,46)=$ $3.86, p<.05$. We will, therefore, partition our analyses by object orientation to investigate the effects of target-object integration on participants' space- and object-based effects.
No other effects were significant in the omnibus analysis (all $F_{\text {S }}<1.71$; see Table 1).

Horizontal orientation We conducted a $2 \times 2$ ANOVA on the mean response latencies in the horizontal condition, with the factors Target-Object Integration ('on' object, 'of' object) and Cue-Target Validity (valid, invalid same-object, invalid different-object). The analysis revealed a significant main effect of cue-target validity, $F(2,46)=42.14, p<.0001$. Planned paired comparisons showed that participants' response latencies were shorter when the target appeared within the valid location $(M=648, S D=82)$ versus the invalid same-object location $(M=713, S D=90), t(23)=7.13, p<.0001$. This analysis also revealed that participants' response latencies were shorter when the target appeared within an invalid same-object location rather than an invalid different-object location $(M=742 \mathrm{~ms}, S D=106), t(23)=4.07, p<.001$. Thus, both space-based and object-based effects emerged when objects were oriented horizontally. Our analysis, however, also revealed a significant interaction between targetobject integration and cue-target validity, $F(2,46)=9.68, p$ $<.0001$. Hence, to investigate the nature of this interaction, we conducted paired $t$ tests separately for each level of targetobject integration in the horizontal condition (see Fig. 10).

Target-object integration and validity Parallel to Experiments 1 and 2, we first calculated difference scores for both the cue validity effects and the same-object advantages, separately for each level of target-object integration. We then conducted planned paired comparisons across those scores for each attentional effect. The magnitudes of both attentional effects differed significantly between levels of target-object integration. The space-based effect was significantly larger when targets were changes 'of' the object $(M=79$ $\mathrm{ms}, S D=69)$ versus 'on' the object $(M=51 \mathrm{~ms}, S D=100)$, $t(23)=2.40, p<.05$. Moreover, the magnitudes of object selection differed significantly between the two targets; the effect was larger when targets were changes 'of' the object $(M=45 \mathrm{~ms}, S D=99)$ versus 'on' the object ( $M=14 \mathrm{~ms}, S D=$ 94), $t(23)=2.88, p<.01$.

Vertical orientation We conducted the same $2 \times 2$ ANOVA on the mean response latencies in the vertical condition. The analysis produced a significant main effect of cue-target validity, $F(2,46)=35.16, p<.0001$. Planned paired comparisons showed that participants' response latencies were shorter when the target appeared within the valid location $(M=628$, $S D=73)$ versus the invalid same-object location $(M=705, S D$ $=93), t(23)=6.43, p<.0001$. Planned paired comparisons also revealed that participants' response latencies were not different when the target appeared within the invalid sameobject versus different-object location $(M=699 \mathrm{~ms}, S D=$ $89), t(23)=0.95, p=.35$. Hence, only a space-based effect 


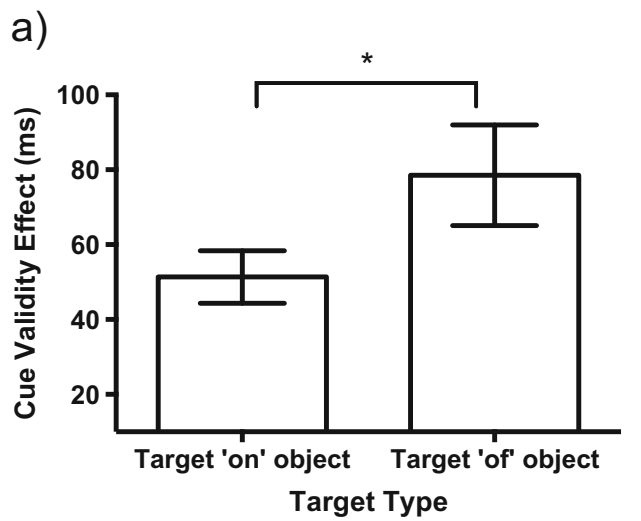

Fig. 10 The (a) space-based and (b) object-based effects for each level of target-object integration in the horizontal condition of Experiment 3. For space-based selection, positive values indicate a cue validity effect, and negative values would indicate a negative cue validity effect. Similarly,

emerged when objects were oriented vertically. However, we also found a significant interaction between target-object integration and cue-target validity, $F(2,46)=6.37, p<.001$. We investigated this two-way interaction by conducting paired $t$ tests separately for each level of target-object integration (see Fig. 11).

Target-object integration and validity We again calculated difference scores for both the space-based and object-based effects, separately for each level of target-object integration. Next, we conducted planned paired comparisons across those scores for each attentional effect. Only the magnitudes of the spatial effect differed significantly between targets. In particular, the space-based effect was significantly larger when the targets were changes 'of' the object $(M=95 \mathrm{~ms}, S D=81)$ versus 'on' the object ( $M=59 \mathrm{~ms}, S D=83), t(23)=3.21, p<$ .01. In contrast, the magnitudes of the object-based effect did not differ significantly between the levels of target-object integration, $t(23)=0.63, p=.54$.

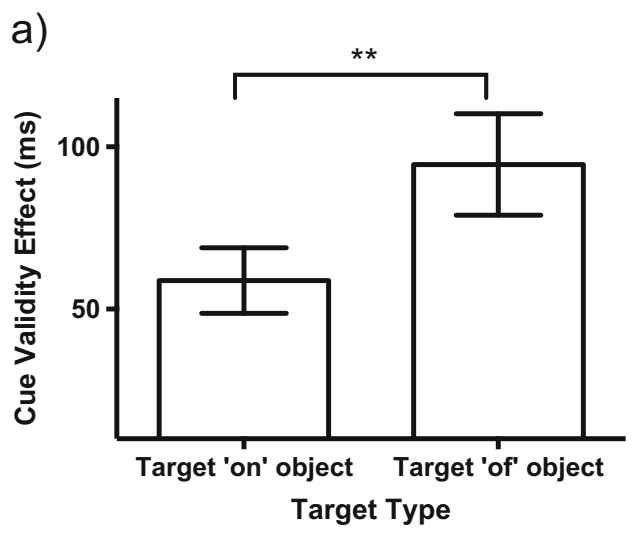

Fig. 11 The (a) space-based and (b) object-based effects for each level of target-object integration in the vertical condition of Experiment 3. For space-based selection, positive values indicate a cue validity effect, and negative values would indicate a negative cue validity effect. Similarly, b)

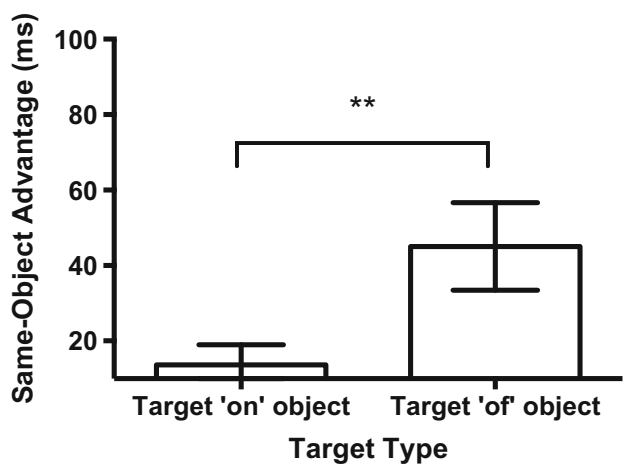

for object-based selection, positive values indicate a same-object advantage, and negative values would indicate a same-object cost. Error bars represent the within-subjects standard errors of the means. ${ }^{*} p<.05,{ }^{* *} p<$ .001

Meridian control analysis We again sought to understand the manner in which object-based attention is allocated along the vertical meridian, but this time when uninformative spatial cues were used. Response latencies were $37 \mathrm{~ms}$ shorter when the target appeared at an invalid same-object location in the vertical orientation rather than at a different-object location in the horizontal orientation, $t(23)=5.62, p<.0001$ (Fig. 6). This finding indicates that, despite the orientation differences we observed, there was still evidence of object-based selection along the vertical meridian when attention was initially oriented exogenously.

\section{Discussion}

The purpose of Experiment 3 was to investigate whether or not the same-object cost in the vertical orientation represented an instance of a meridian effect that was driven by endogenous orienting; that is, we examined whether the discrepancy in the efficiencies of object-based attention allocation across meridians (whereby crossing the vertical meridian appeared

b)

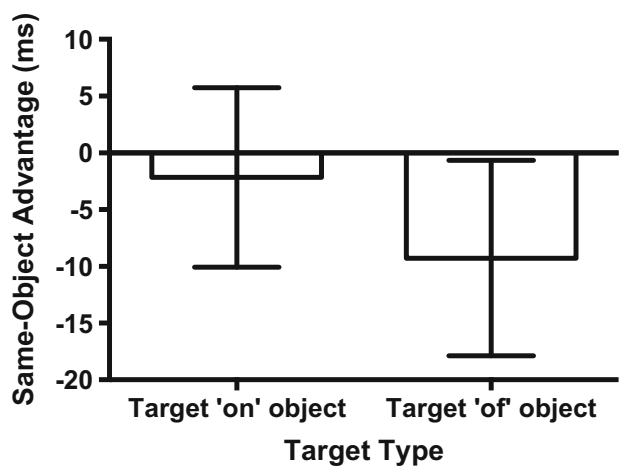

for object-based selection, positive values indicate a same-object advantage, and negative values indicate a same-object cost. Error bars represent the within-subjects standard errors of the means. ${ }^{* *} p<.01$ 
more efficient than crossing the horizontal meridian) was an outcome of the mode of attentional orienting. Thus, to examine this question we encouraged participants to shift spatial attention exogenously (rather than endogenously or in hybrid form, as in the prior experiments) by utilizing uninformative peripheral cues. Experiment 3 revealed two sets of results: (a) The magnitudes of the same-object advantage differed between levels of target-object integration; the effect was larger when targets appeared as part 'of' versus 'on' a horizontal object. This result differed from the results of Experiment 2 (in which target-object integration did not modulate objectbased selection), but mirrored the results of Experiment 1 (in which target-object integration did modulate object-based selection). Thus, the effect of target-object integration on object-based selection appears only when the target location is uncertain (as in Exps. 1 and 3), but not when it is largely known (as in Exp. 2, in which the target appeared in the cued location on $75 \%$ of trials). (b) We found no evidence of the previously observed same-object cost in the vertical orientation, and we continued to find evidence of object-based effects along the vertical meridian when parceling out shifts across the vertical meridian. ${ }^{3}$ To our knowledge, this is the first time that a meridian effect (masked as a same-object cost in the vertical orientation) has been shown in an object-based attention task, and later ameliorated by encouraging exogenous spatial orienting. It is also worth mentioning that the difference in the sizes of the spatial effect between the vertical and horizontal orientations, which had been significant in Experiment $2(M=38 \mathrm{~ms}, S D=86), t(23)=4.24, p<.001$, was no longer significant $(M=12 \mathrm{~ms}, S D=37), t(23)=1.57$, $p=.13$. This result suggests that exogenous orienting also ameliorated the extent to which crossing the horizontal meridian affected the allocation of spatial attention. Note that exogenous orienting merely reduced the effect of meridians on object-based selection, thus indicating that the control of spatial attention is not the only factor driving meridian costs.

\section{General discussion}

Various studies have shown that object-based effects are less robust than space-based effects (for a review, see Reppa, Schmidt, \& Leek, 2012). Unsurprisingly, these variations in object-based effects, as compared to space-based effects,

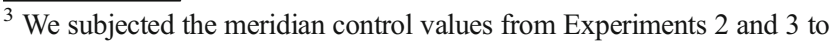
an independent-samples $t$ test and found no significant difference between experiments, $t(46)=1.91, p=.06$. This result suggests that the addition of an uninformative spatial cue did not enhance object-based selection along the vertical meridian in a manner that was significantly different from a hybrid spatial cue. It is important to also mention that the meridian control values in Experiment 1 did not differ from those in Experiments 2 and 3 (all $p \mathrm{~s}>.63$ ), which suggests that the magnitudes of object selection along the vertical meridian were unaffected by our manipulations and, thus, were comparable across participants and experiments.
}

across paradigms have raised important questions about the conditions that give rise to, or modulate, object-based selection. The purpose of the present study was, therefore, to investigate the contributions of three factors to object-based selection: target-object integration, attention distribution, and object orientation. We examined these specific factors because prior research had indicated that they may account for variations in object-based effects (e.g., Lamy \& Egeth, 2002; Pilz et al., 2012). We conducted this investigation by presenting participants with both the double-rectangle cueing paradigm and the wrenches paradigm, while manipulating, systematically, the task characteristics (object orientation and distribution of attention).

We observed several important findings in this study with regard to the roles of target-object integration and attention distribution in object-based selection. First, we found that object-based selection occurred under conditions of wide attention allocation only when targets were changes 'of' objects versus 'on' objects (Exp. 1). Note that this result does not indicate that object-based selection is limited to strongly integrated targets and objects when attention is initially focused widely (indeed, Goldsmith \& Yeari, 2003, and Greenberg, 2009 , both showed that weakly integrated targets and objects produce object-based selection effects under such conditions). Rather, this aspect of our findings replicates the pattern of results observed by Lamy and Egeth (2002), insofar as it suggests that object selection-when targets and objects are integrated weakly (i.e., when targets appear floating 'on' an object), but not when targets and objects are integrated strongly (i.e., when targets appear as part 'of' an object)_benefits from an initial narrowing of attention. This initial narrowing of attention may jump-start object selection when targets and objects are integrated weakly, either because it enables the occurrence of attention shifts from one possible target location to another or because it enables prioritization of the cued object; both of these strategies may be sufficient for the allocation of object-based attention under such conditions. Second, and confirming this suggestion, we found a same-object advantage under conditions of narrow attention allocation for both levels of target-object integration (Exps. 2 and 3). Importantly, however, the same-object advantage under conditions of narrow attention allocation was larger in magnitude for targets that were changes 'of' objects versus 'on' objects, when target location was less certain (Exp. 3). This aspect of our findings extends the pattern of results observed by Lamy and Egeth, insofar as it shows that the effect of target-object integration on object-based selection not only emerges when attention is focused widely, but also when focusing attention narrowly is not useful. That is, under conditions in which the cue is uninformative of the target location, object-based selection is "enhanced" when targets and objects are integrated strongly, but not when they are integrated weakly. Of course, our manipulations of attentional distribution were conducted 
between subjects, and thus it is possible that our observation that target-object integration modulates object selection differently under conditions of wide versus narrow attention focusing was affected by each group of participants' perceptions of the degree to which the targets and objects were integrated (particularly in the target 'of' object case). We recommend that future studies carry out such manipulations within the same group of participants. Nonetheless, our findings suggest that the strength of target-object integration may affect the strategies that participants adopt to allocate object-based attention, consistent with previous proposals of strategic control of object-based attention (Drummond \& Shomstein, 2010; Greenberg, 2009; Greenberg \& Gmeindl, 2008).

Object-based selection is commonly attributed to an attentional prioritization mechanism or strategy (Shomstein \& Yantis, 2002, 2004), whereby priority maps guide participants' search for a target (Drummond \& Shomstein, 2010; Sentürk, Greenberg, \& Liu, 2016). This proposal posits that the same-object advantage emerges because unattended regions of an attended object are assigned higher priority than unattended regions of an unattended object. The strategy of ordering the processing priority of objects to find a target is, however, abandoned (and object-based effects do not emerge) when the target location is certain (cf. Drummond \& Shomstein, 2010; Greenberg, 2009). Our results constrain this attentional-prioritization hypothesis by indicating that participants rely more on priority maps to guide attentional selection under conditions of target uncertainty (e.g., Exp. 3) when the strength of the object representation is low versus high (as indexed by the strength of target-object integration). In contrast, participants utilize priority maps to the same degrees when the strength of the object representation is low versus high under conditions of target certainty (e.g., Exp. 2). Of importance, this caveat to the attentional prioritization account implies that the strategy that participants use to prioritize objects is not carried out by default but, rather, invokes top-down control. Note that our findings cannot be reconciled with the proposal that object-based effects are due to a spreading of attention throughout an attended object, because that view necessitates a reflexive (rather than top-down) spread of attention, irrespective of target location certainty (e.g., Richard, Lee, \& Vecera, 2008). ${ }^{4}$

Our results also help clarify the role of object orientation on object-based selection. First, we found that the space-based

\footnotetext{
${ }^{4}$ It is important to acknowledge that participants are encouraged to encode the locations of the targets as being somewhat separate from the objects in the double-rectangle paradigm, due to abrupt onsets (see, e.g., Yantis \& Jonides, 1984). Theoretically, this strategy may have implications for the prioritization theory of object-based attention, because it posits that specific target locations (not the objects themselves) guide the flexible prioritization of object-based selection. The prioritization theory may, therefore, hold mainly (or only) in cases wherein targets and objects are weakly integrated (specifically, when targets appear 'on' the objects).
}

effect was larger when objects were oriented vertically versus horizontally (Exp. 2). This effect indicates that space-based attention is more easily reallocated when crossing the vertical than the horizontal meridian, which supports prior findings by Carrasco, McElree, and Giordano (2002). Second, we found a same-object advantage when objects were oriented horizontally, but a same-object cost when objects were oriented vertically (Exps. 1 and 2). This effect indicates that object-based attention is also more easily allocated when crossing the vertical meridian as opposed to the horizontal meridian (see also de-Wit et al., 2009; Pilz et al., 2012). We interpreted these modulations of space- and object-based attention as evidence of a meridian effect, which emerges due to anisotropies in the cortical representations when spatial attention is oriented endogenously (e.g., Klein \& Pontefract, 1991). We refer the reader to a detailed exploration of the meridian effect in the context of object-based attention in the present issue of this journal (Barnas \& Greenberg, 2016). Third, and in confirmation of this hypothesis, we found that the effects of object orientation on space- and object-based selection are ameliorated when participants shift attention purely exogenously (Exp. 3). This finding shows that modulations in both space- and object-based effects by object orientation can be attributed to an inhomogeneity in performance across the visual field. It is worth noting that ameliorations of both space- and object-based meridian effects are surprising, because participants were only encouraged to orient spatial, but not object-based, attention exogenously. Indeed, it is unclear whether or not encouraging pure object-based attentional orienting would also ameliorate the effect of object orientation on space- and object-based selection (i.e., the meridian effect). This issue, to our knowledge, has not yet been examined. Fourth, and consistent with the latter finding, we found evidence of objectbased selection along the vertical meridian (when parceling out shifts across the vertical meridian) in all experiments. This finding shows that the effect of orientation on objectbased selection is likely an artifact caused by the effects of shifting attention across the meridians.

Of course, these findings beg the following question: For what reason is selective attention (both space- and objectbased) re/allocated more efficiently when crossing the vertical than when crossing the horizontal meridian? That is, what is the nature of these artifact-inducing meridian effects? At first glance our findings seem surprising, given the contralateral organization of the visual system, which should serve to impair (rather than facilitate) attentional shifts crossing the vertical meridian versus the horizontal meridian (Reuter-Lorenz \& Fendrich, 1992; but see Holtzman, Sidtis, Volpe, Wilson, \& Gazzaniga, 1981). It is known, of course, that the lower and upper visual field representations of retinotopic areas within extrastriate cortex are sequestered dorsally and ventrally, respectively (Van Essen, 1985). Hence, although the horizontal 
meridian does not represent an interhemispheric barrier in the same way as the vertical meridian, it does represent an intrahemispheric barrier (Sereno \& Kosslyn, 1991). In correspondence with this theory of an attentional advantage when crossing the vertical versus the horizontal meridian, other research has shown that visual performance is superior across the vertical meridian because discriminability is comparatively enhanced and temporal dynamics are comparatively rapid (although the rate of information accrual may be faster along the disadvantaged meridian; Carrasco et al., 2002). Indeed various studies have shown that visual acuity (Beirne, Zlatkova, \& Anderson, 2005; Rovamo, Virsu, Laurinen, \& Hyvarinen, 1982) and contrast sensitivity (Carrasco, Evert, Chang, \& Katz, 1995; Carrasco, Talgar, \& Cameron, 2001; Rijsdijk, Kroon, \& van der Wildt, 1980) are better when crossing the vertical versus the horizontal meridian. Importantly, the presence of the meridian effect in object-based attention underscores the importance of including Orientation as a factor when analyzing data, which is surprisingly atypical across studies in this field (e.g., Goldsmith \& Yeari, 2003; He, Fan, Zhou, \& Chen, 2004; Lamy \& Egeth, 2002; Marino \& Scholl, 2005; Moore et al., 1998). Alternatively, researchers could use the method included herein to calculate object-based effects while controlling for shifts of attention across the meridians.

\section{Conclusion}

The purpose of the present study was to examine three factors that may drive variations in object-based effects: target-object integration, attention distribution, and object orientation. We found that the strength of target-object integration is important for object-based selection, such that it can affect the occurrence of object-based selection when attention is initially focused widely, and the magnitude of object-based selection when the target location is uncertain. We also found that object orientation is important for object-based selection, such that it can affect the sign of object-based selection when attention is oriented endogenously. These results imply that variations in object-based effects do not occur when the factors TargetObject Integration, Attention Distribution, and Object Orientation are present alone, but, rather, when they are present in combination. Future studies can, hence, utilize this information about the boundary conditions of object-based attention to formulate hypotheses about the mechanisms that underlie such selection.

Author note We thank Rachel Bogatay, Jonathan Reich, and Mary Liz Kim for help with data collection. This work was supported by US-Israel Binational Science Foundation Grant Number 2013400 (to A.S.G.).

\section{References}

Abrams, R. A., \& Law, M. B. (2000). Object-based visual attention with endogenous orienting. Perception \& Psychophysics, 62, 818-833. doi:10.3758/BF03206925

Barnas, A. J., \& Greenberg, A. S. (2016). Visual field meridians modulate the re-allocation of object-based attention. Attention, Perception, \& Psychophysics, 78, doi:10.3758/s13414-016-1116-5.

Behrmann, M., Zemel, R. S., \& Mozer, M. C. (1998). Object-based attention and occlusion: Evidence from normal participants and a computational model. Journal of Experimental Psychology: Human Perception and Performance, 24, 1011-1036. doi:10. 1037/0096-1523.24.4.1011

Beirne, R. O., Zlatkova, M. B., \& Anderson, R. S. (2005). Changes in human short-wavelength-sensitive and achromatic resolution acuity with retinal eccentricity and meridian. Visual Neuroscience, 22, 7986. doi:10.1017/S0952523805221119

Berlucchi, G., di Stefano, M., Marzi, C. A., Morelli, M., \& Tassinari, G. (1981). Direction of attention in the visual field as measured by a reaction time paradigm. Behavioural Brain Research, 2, 244-245.

Berlucchi, G., Tassinari, G., Marzi, C. A., \& di Stefano, M. (1989). Spatial distribution of the inhibitory effect of peripheral noninformative cues on simple reaction time to non-fixated visual targets. Neuropsychologia, 27, 201-221. doi:10.1016/0028-3932(89) 90172-3

Botta, F., Santangelo, V., Raffone, A., Lupiáñez, J., \& Belardinelli, M. O. (2010). Exogenous and endogenous spatial attention effects on visuospatial working memory. Quarterly Journal of Experimental Psychology, 63, 1590-1602. doi:10.1080/17470210903443836

Carrasco, M., Evert, D. L., Chang, I., \& Katz, S. M. (1995). The eccentricity effect: Target eccentricity affects performance on conjunction searches. Perception \& Psychophysics, 57, 1241-1261. doi:10. 3758/BF03208380

Carrasco, M., McElree, B., \& Giordano, A. (2002, May). Covert attention speeds information accrual along the vertical than the horizontal meridian. Paper presented at the Vision Sciences Society conference, Saratosa, FL.

Carrasco, M., Talgar, C. P., \& Cameron, E. L. (2001). Characterizing visual performance fields: Effects of transient covert attention, spatial frequency, eccentricity, task and set size. Spatial Vision, $15,61-75$.

Cepeda, N. J., \& Kramer, A. F. (1999). Strategic effects on object-based attentional selection. Acta Psychologica, 103, 1-19. doi:10.1016/ S0001-6918(99)00021-9

Chen, H.-C., \& Huang, L. (2015). Solving the paradox between sameobject advantage and different-object advantage. Vision Research, $115,128-134$.

Conci, M., \& Müller, H. J. (2009). The "beam of darkness": Spreading of the attentional blink within and between objects. Attention, Perception, \& Psychophysics, 71, 1725-1738. doi:10.3758/APP. 71.8.1725

Davis, G., \& Holmes, A. (2005). Reversal of object-based benefits in visual attention. Visual Cognition, 12, 817-846. doi:10.1080/ 13506280444000247

Davis, G., Welch, V. L., Holmes, A., \& Shepherd, A. (2001). Can attention select only a fixed number of objects at a time? Perception, 30, 1227-1248. doi:10.1068/P3133

de-Wit, L. H., Kentridge, R. W., \& Milner, A. D. (2009). Object-based attention and visual area LO. Neuropsychologia, 47, 1483-1490. doi:10.1016/j.neuropsychologia.2008.11.002

Downing, C. J., \& Pinker, S. (1985). The spatial structure of visual attention. Hillsdale, NJ: Erlbaum.

Drummond, L., \& Shomstein, S. (2010). Object-based attention: Shifting or uncertainty? Attention, Perception, \& Psychophysics, 72, 17431755. doi:10.3758/APP.72.7.1743 
Duncan, J. (1984). Selective attention and the organization of visual information. Journal of Experimental Psychology: General ,113(4), 501-517. doi:10.1037/0096-3445.113.4.501

Egly, R., Driver, J., \& Rafal, R. D. (1994). Shifting visual-attention between objects and locations-Evidence from normal and parietal lesion subjects. Journal of Experimental Psychology: General, 123, 161-177. doi:10.1037/0096-3445.123.2.161

Goldsmith, M., \& Yeari, M. (2003). Modulation of object-based attention by spatial focus under endogenous and exogenous orienting. Journal of Experimental Psychology: Human Perception and Performance, 29, 897-918. doi:10.1037/0096-1523.29.5.897

Greenberg, A. S. (2009). Uncertainty as a guiding principle in the strategic allocation of attention to objects (Ph.D. dissertation). Johns Hopkins University, Baltimore, MD.

Greenberg, A. S., \& Gmeindl, L. (2008). Strategic control of attention to objects and locations. Journal of Neuroscience, 28, 564-565. doi: 10.1523/JNEUROSCI.4386-07.2008

Greenberg, A. S., Hayes, D., Roggeveen, S., Creighton, P., Bennett, A., Sekuler, K., \& Pilz, K. S. (2014). Object-based attention is modulated by shifts across the meridians [Abstract]. Journal of Vision, 14(10), 1062a. doi:10.1167/14.10.1062

Greenberg, A. S., Rosen, M., Cutrone, E., \& Behrmann, M. (2015). The effects of visual search efficiency on object-based attention. Attention, Perception, \& Psychophysics, 77, 1544-1557. doi:10. 3758/s13414-015-0892-7

Harrison, S. J., \& Feldman, J. (2009). Perceptual comparison of features within and between objects: A new look. Vision Research, 49, 2790 2799. doi:10.1016/j.visres.2009.08.014

He, X., Fan, S., Zhou, K., \& Chen, L. (2004). Cue validity and objectbased attention. Journal of Cognitive Neuroscience, 16, 1085-1097. doi:10.1162/0898929041502689

Holtzman, J. D., Sidtis, J. J., Volpe, B. T., Wilson, D. H., \& Gazzaniga, M. S. (1981). Disassociation of spatial information for stimulus localisation and the control of attention. Brain, 104, 861-872.

Hughes, H. C., \& Zimba, L. D. (1987). Natural boundaries for the spatial spread of directed visual-attention. Neuropsychologia, 25, 5-18. doi:10.1016/0028-393290039-X

Klein, R. M., \& Pontefract, A. (1991). Does oculomotor readiness mediate cognitive control of visual attention? Revisited! Cambridge, MA: MIT Press.

Lamy, D., \& Egeth, H. (2002). Object-based selection: The role of attentional shifts. Perception \& Psychophysics, 64, 52-66. doi:10.3758/ BF03194557

Lavie, N., \& Driver, J. (1996). On the spatial extent of attention in objectbased visual selection. Perception \& Psychophysics, 58, 1238 1251. doi:10.3758/Bf03207556

Law, M. B., \& Abrams, R. A. (2002). Object-based selection within and beyond the focus of spatial attention. Perception \& Psychophysics, 64, 1017-1027. doi:10.3758/BF03194753

Macquistan, A. D. (1997). Object-based allocation of visual attention in response to exogenous, but not endogenous, spatial precues. Psychonomic Bulletin \& Review, 4, 512-515. doi:10.3758/ Bf03214341

Marino, A. C., \& Scholl, B. J. (2005). The role of closure in defining the "objects" of object-based attention. Perception \& Psychophysics, 67, 1140-1149. doi:10.3758/BF03193547

Moore, C. M., Yantis, S., \& Vaughan, B. (1998). Object-based visual selection: Evidence from perceptual completion. Psychological Science, 9, 104-110. doi:10.1111/1467-9280.00019

Neill, W. T., Li, Y., Seror, G., \& O'Connor, P. (2009). Between-object superiority in divided attention [Abstract]. Journal of Vision, 9(8), 207a. doi:10.1167/9.8.207
Pilz, K. S., Roggeveen, A. B., Creighton, S. E., Bennett, P. J., \& Sekuler, A. B. (2012). How prevalent is object-based attention? PLoS ONE, 7, e30693. doi:10.1371/journal.pone.0030693

Posner, M. I., \& Cohen, Y. (1984). Components of visual orienting. In H. Bouma \& D. G. Bouwhuis (Eds.), Attention and performance X: Control of language processes (pp. 531-556). Hillsdale, NJ: Erlbaum.

Reppa, I., Schmidt, W. C., \& Leek, E. C. (2012). Successes and failures in producing attentional object-based cueing effects. Attention, Perception, \& Psychophysics, 74, 43-69. doi:10.3758/s13414-0110211-x

Reuter-Lorenz, P. A., \& Fendrich, R. (1992). Oculomotor readiness and covert orienting-Differences between central and peripheral precues. Perception \& Psychophysics, 52, 336-344. doi:10.3758/ BF03209150

Richard, A. M., Lee, H., \& Vecera, S. P. (2008). Attentional spreading in object-based attention. Journal of Experimental Psychology: Human Perception and Performance, 34, 842-853. doi:10.1037/ 0096-1523.34.4.842

Rijsdijk, J. P., Kroon, J. N., \& van der Wildt, G. J. (1980). Contrast sensitivity as a function of position on the retina. Vision Research, 20, 235-241.

Rizzolatti, G., Riggio, L., Dascola, I., \& Umiltà, C. (1987). Reorienting attention across the horizontal and vertical meridians-Evidence in favor of a premotor theory of attention. Neuropsychologia, 25, 3140. doi:10.1016/0028-3932(87)90041-8

Rovamo, J., Virsu, V., Laurinen, P., \& Hyvarinen, L. (1982). Resolution of gratings oriented along and across meridians in peripheral vision. Investigative Ophthalmology \& Visual Science, 23, 666-670.

Sentürk, G., Greenberg, A. S., \& Liu, T. (2016). Saccade latency indexes exogenous and endogenous object-based attention. Attention, Perception, \& Psychophysics, 78, doi:10.3758/s13414-016-1136-1.

Sereno, A. B., \& Kosslyn, S. M. (1991). Discrimination within and between hemifields - A new constraint on theories of attention. Neuropsychologia, 29, 659-675. doi:10.1016/0028-3932(91) 90100-M

Shomstein, S., \& Behrmann, M. (2008). Object-based attention: Strength of object representation and attentional guidance. Perception \& Psychophysics, 70, 132-144. doi:10.3758/PP.70.1.132

Shomstein, S., \& Yantis, S. (2002). Object-based attention: Sensory modulation or priority setting? Perception \& Psychophysics, 64, 41-51. doi:10.3758/BF03194556

Shomstein, S., \& Yantis, S. (2004). Control of attention shifts between vision and audition in human cortex. Journal of Neuroscience, 24, 10702-10706. doi:10.1523/JNEUROSCI.2939-04.2004

Tassinari, G., Aglioti, S., Chelazzi, L., Marzi, C. A., \& Berlucchi, G. (1987). Distribution in the visual-field of the costs of voluntarily allocated attention and of the inhibitory aftereffects of covert orienting. Neuropsychologia, 25, 55-71. doi:10.1016/00283932(87)90043-1

Van Essen, D. (1985). Functional organisation of primate visual cortex (Vol. 3). New York, NY: Plenum Press.

Watson, A. B., \& Pelli, D. G. (1983). QUEST: A Bayesian adaptive psychometric method. Perception \& Psychophysics, 33, 113-120. doi:10.3758/BF03202828

Watson, S. E., \& Kramer, A. F. (1999). Object-based visual selective attention and perceptual organization. Perception \& Psychophysics, 61, 31-49. doi:10.3758/BF03211947

Yantis, S., \& Jonides, J. (1984). Abrupt visual onsets and selective attention: Evidence from visual search. Journal of Experimental Psychology: Human Perception and Performance, 10, 601-621. doi:10.1037/0096-1523.10.5.601 